
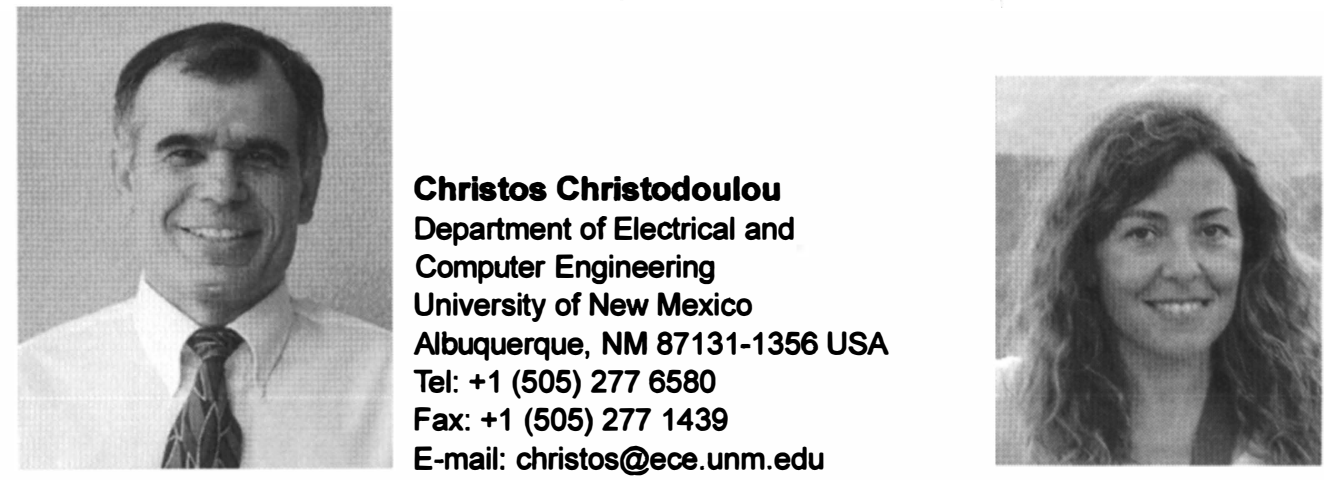

\section{Eva Rajo-Iglesias}

Departamento de Teoría de la Señal y Comunicaciones University Carlos III of Madrid, Despacho 4.3B10

Avenida de la Universidad, 30, 28911 Leganés, Madrid, Spain Tel: +34 916248774

Fax: +34 916248749

E-mail: eva@tsc.uc3m.es

\title{
On the Capacity of Printed Planar Rectangular Patch Antenna Arrays in the MIMO Channel: Analysis and Measurements
}

\author{
Celal Alp Tunc ${ }^{1}$, Ugur Olgun ${ }^{2}$, Vakur B. Ertürk ${ }^{3}$, and Ayhan Altintas ${ }^{3}$ \\ ${ }^{1}$ Ankara Research and Technology Laboratory (ARTLab Ltd) \\ Ankara, Turkey \\ ${ }^{2}$ The Ohio State University, ElectroScience Laboratory \\ Columbus, OH 43212 USA \\ ${ }^{3}$ Department of Electrical and Electronics Engineering \\ Bilkent University \\ Bilkent, Ankara, TR-06800, Turkey \\ E-mail: alp.tunc@artlab.com.tr
}

\begin{abstract}
Printed arrays of rectangular patch antennas are analyzed in terms of their MIMO performance using a full-wave channel model. These antennas are designed and manufactured in various array configurations, and their MIMO performance is measured in an indoor environment. Good agreement is achieved between the measurements and simulations performed using the full-wave channel model. Effects on the MIMO capacity of the mutual coupling and the electrical properties of the printed patches, such as the relative permittivity and thickness of the dielectric material, are explored.
\end{abstract}

Keywords: MIMO systems; antenna arrays; microstrip antennas; microstrip arrays; planar printed arrays; antenna array mutual coupling; moment methods 


\section{Introduction}

$\mathbf{M}$ icrostrip patch antennas have gained immense popularity in military and commercial applications, owing to their conformal, lightweight, and low cost nature. Moreover, the recent decline of low-loss dielectric substrate prices has boosted their utilization, particularly in commercial communications applications. Their performance in multiple-input multiple-output (MIMO) systems thus seems very promising [1-8], but has to be investigated in detail.

In [9], the MIMO performance of printed dipole arrays was analyzed using a full-wave channel model, based on the Method of Moments (MoM) solution of the electric-field integral equation (EFIE). Comparisons with freestanding dipoles were given in terms of the channel capacity. Effects of the electrical properties of printed dipoles on the MIMO capacity were explored in terms of the relative permittivity and thickness of the dielectric material.

In this work, the study on printed dipoles [9] is expanded by modifying the full-wave channel model developed before for analyzing probe-fed rectangular patches. Several probe-fed rectangular microstrip patches are fabricated and employed in a MIMO wireless communication system. The performance of different patch antenna arrays in terms of MIMO channel capacity is measured and the results are compared with each other, as well as the numerical results of the full-wave channel model.

\section{Wireless Channel Measurement Using a Vector Network Analyzer}

Let us consider the wireless communication system with single antennas at the transmitter (TX) and receiver (RX) sides as illustrated in Figure 1. Using network analysis, the total port voltages and currents at the terminal planes for the transmitter port (port 1) and the receiver port (port 2) can be written as

$$
\begin{aligned}
& V_{n}=\left(a_{n}+b_{n}\right) \sqrt{Z_{i n, n}}, \\
& I_{n}=\frac{a_{n}-b_{n}}{\sqrt{Z_{i n, n}}},
\end{aligned}
$$

where $n=1,2 . Z_{i n, n}$ is the input impedance seen through the port, and is taken equal to the characteristic impedance of the port, $Z_{0, n}$. $a_{n}$ represents an incident wave at the $n$th port, and $b_{n}$ represents a wave reflected from that port. These inward and outward propagating waves are related to each other via the scattering $(S)$ parameters:

$$
\begin{aligned}
& \bar{b}=\mathbf{S} \bar{a}, \\
& {\left[\begin{array}{l}
b_{1} \\
b_{2}
\end{array}\right]=\left[\begin{array}{ll}
s_{11} & s_{12} \\
s_{21} & s_{22}
\end{array}\right]\left[\begin{array}{l}
a_{1} \\
a_{2}
\end{array}\right] .}
\end{aligned}
$$

The voltage and the current at the transmitting-antenna port then become

$$
V_{1}=\left(a_{1}+s_{11} a_{1}+s_{12} a_{2}\right) \sqrt{Z_{i n, 1}} \text {, }
$$

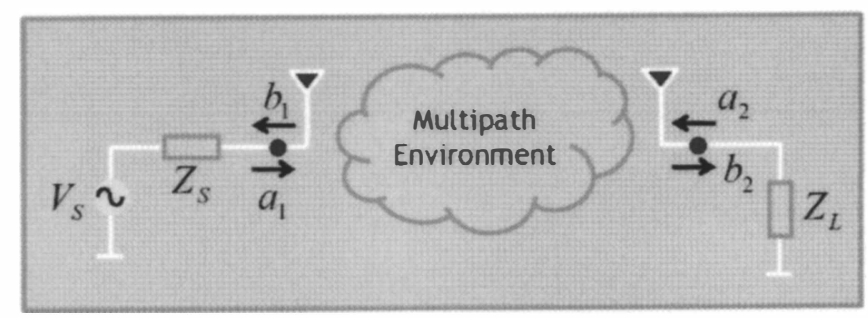

Figure 1. A SISO wireless communication system.

$$
I_{1}=\frac{a_{1}-s_{11} a_{1}-s_{12} a_{2}}{\sqrt{Z_{i n, 1}}} .
$$

The source voltage $\left(V_{S}\right)$ can be expressed in terms of the source impedance $\left(Z_{S}\right)$, the port voltage, and the current by

$$
V_{S}=V_{1}+I_{1} Z_{S}
$$

If the input impedance of the transmitting antenna is matched to the source impedance, such that $Z_{i n, 1}=Z_{S}=Z_{0,1}$, Equation (7) turns out to be

$$
V_{S}=2 a_{1} \sqrt{Z_{i n, 1}}
$$

Similarly, when the input impedance of the receiving antenna is matched to the load impedance $Z_{i n, 2}=Z_{L}=Z_{0,2}$, the following relations are valid:

$$
\begin{aligned}
V_{2} & =\left(a_{2}+b_{2}\right) \sqrt{Z_{i n, 2}}=-I_{2} Z_{L} \\
& =-\left(a_{2}-b_{2}\right) \sqrt{Z_{i n, 2}} .
\end{aligned}
$$

The equality in Equation (9), taken with Equation (10), yields

$$
a_{2}=0
$$

and

$$
V_{L}=b_{2} \sqrt{Z_{i n, 2}}
$$

Assuming $Z_{S}=Z_{L}$, the channel response defined from the source voltage to the load voltage can be expressed as

$$
h=\frac{V_{L}}{V_{S}}=\frac{1}{2} \frac{b_{2}}{a_{1}},
$$

which becomes

$$
h=\frac{s_{21}}{2}
$$

since $a_{2}=0$

Namely, if the input impedances of the transmitting and receiving antennas are matched, $Z_{i n, 1}=Z_{i n, 2}=Z_{S}=Z_{L}$, measuring $S_{21}$ of such a communication system with the use of a two- 
Table 1. The dielectric-substrate parameters.

\begin{tabular}{|c|c|c|c|}
\hline Antenna & A & B & C \\
\hline$\varepsilon_{r}$ & 3.0 & 3.2 & 4.5 \\
\hline $\tan \delta$ & 0.040 & 0.045 & 0.030 \\
\hline$d(\mathrm{~mm})$ & 1.524 & 0.508 & 1.575 \\
\hline
\end{tabular}

Table 2. The parameters of the rectangular patches.

\begin{tabular}{|c|c|c|c|}
\hline Antenna & A & B & C \\
\hline$W$ & $0.353 \lambda$ & $0.339 \lambda$ & $0.301 \lambda$ \\
\hline$L$ & $0.283 \lambda$ & $0.281 \lambda$ & $0.232 \lambda$ \\
\hline$F P(x, y)$ & $0.089 \lambda, W / 2$ & $0.209 \lambda, W / 2$ & $0.156 \lambda, W / 2$ \\
\hline
\end{tabular}

port vector network analyzer (VNA) will give twice the channel response, since the impedances seen through the ports are usually equal $\left(Z_{S}=Z_{L}=50 \Omega\right)$. Vector network analyzer systems have therefore frequently been used to measure the channel characteristics of indoor environments [10-12]. For the measurement of the channel-matrix entries of a $2 \times 2$ MIMO system, either a four-port vector network analyzer alone, or a two-port vector network analyzer with appropriate switches, can be used [10].

\section{Design and Production of the Patch Antenna Arrays}

Three sets of low-loss dielectric substrates with copper on both sides were chosen, the characteristic parameters of which are given in Table 1 . In Table $1, \varepsilon_{r}$ and $d$ represent the dielectric permittivity and thickness, respectively. Furthermore, $\tan \delta=\sigma /\left(\omega \varepsilon_{0} \varepsilon_{r}\right)$ is the loss tangent, which represents the loss due to the conductivity $(\sigma)$.

In [13], analytical expressions were given for the input impedance in terms of the characteristic parameters of the rectangular patch antenna, such as the width, length, dielectric thickness, permittivity, and feed-point coordinates, as shown in Figure 2. By employing these expressions, coax-fed rectangular patch antennas with $50 \Omega$ input impedances were designed to operate at $f=1.9725 \mathrm{GHz}$. The designs were then verified by a commercially available electromagnetic solver, based on the mixed-potential integral equation. They were carefully fabricated to make sure that all antennas were matched to $50 \Omega$ at the operating frequency. Figure 3 shows curves of the measured $\left|S_{11}\right|$ as a function of frequency for the fabricated antennas $\mathrm{A}, \mathrm{B}$, and $\mathrm{C}$. The final parameters of these antennas (i.e., the width $(W)$, the length $(L)$, and the coordinates of the feed points $[F P(x, y)]$ ) are tabulated in Table 2 . In Table $2, \lambda$ represents the free-space wavelength. The simulated radiation-field patterns of all three antennas are also illustrated in Figure 4.

Arrays with various configurations were fabricated next. For each substrate type, mainly two-element arrays were manufactured, the antennas of which were located in side-by-side $(2 \times 1)$ or collinear $(1 \times 2)$ arrangements. To investigate the effects of mutual coupling on the MIMO channel capacity, antenna elements in an array were sited either close to (C), or far away (F) from, each

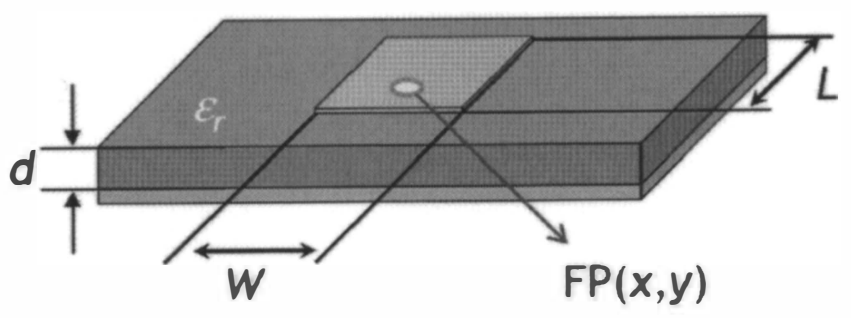

Figure 2. The geometry of a rectangular patch antenna.

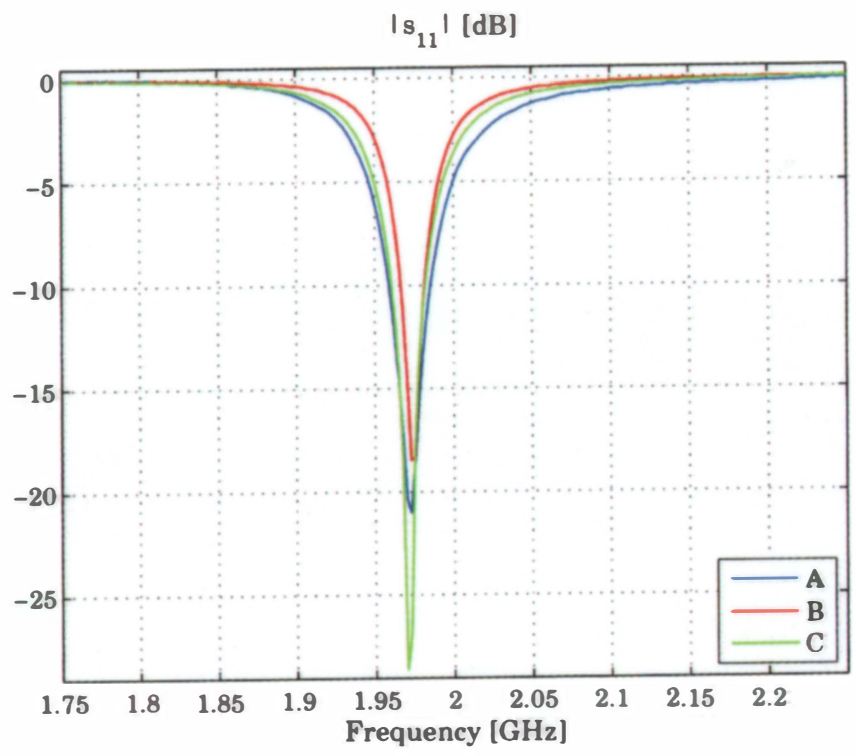

Figure 3. The measured $\left|S_{11}\right|$ (in $\mathbf{d B}$ ) as a function of frequency for the fabricated antennas $(\mathrm{A}, \mathrm{B}, \mathrm{C})$.

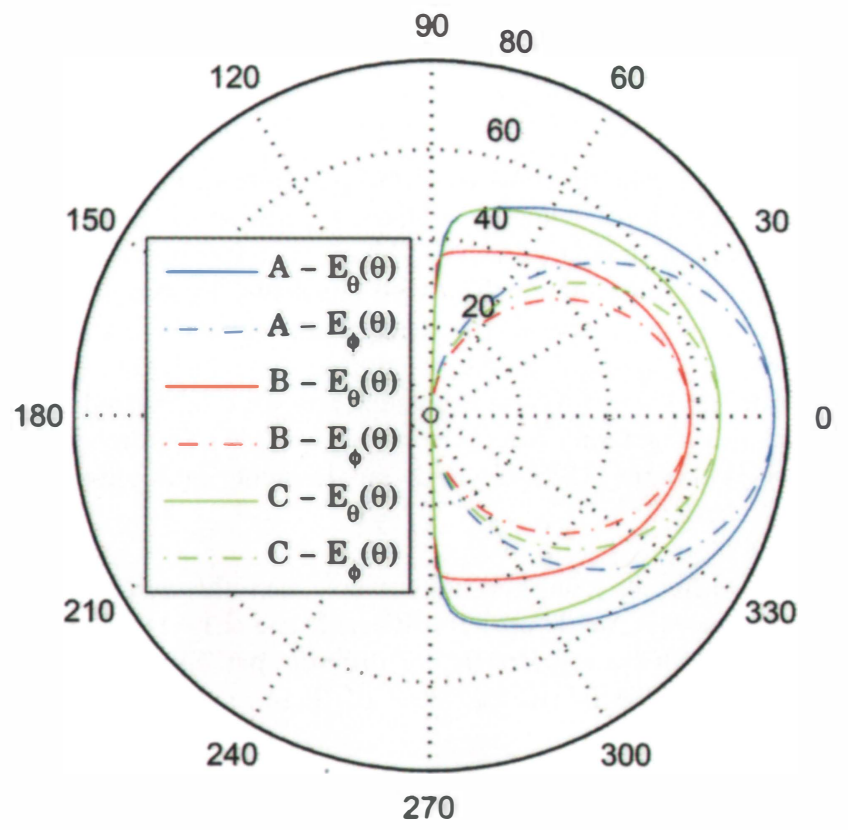

Figure 4. The simulated radiation patterns of antennas $A, B$, and $C$ (the electrical and geometrical parameters of antennas $A, B$, and $C$ are given in Tables 1 and 2 ). 
Table 3. The fabricated patch array configurations with the distances between the feed points $(\Delta / \lambda)$.

\begin{tabular}{|c|c|}
\hline Antenna & $\Delta / \lambda$ \\
\hline $\mathrm{A} 1 \times 2 \mathrm{C}$ & 0.403 \\
\hline $\mathrm{A} 1 \times 2 \mathrm{~F}$ & 0.800 \\
\hline $\mathrm{A} 2 \times 1 \mathrm{C}$ & 0.333 \\
\hline $\mathrm{A} 2 \times 1 \mathrm{~F}$ & 0.800 \\
\hline $\mathrm{B} 1 \times 2 \mathrm{C}$ & 0.388 \\
\hline $\mathrm{B} 1 \times 2 \mathrm{~F}$ & 0.800 \\
\hline $\mathrm{B} 2 \times 1 \mathrm{C}$ & 0.331 \\
\hline $\mathrm{B} 2 \times 1 \mathrm{~F}$ & 0.800 \\
\hline $\mathrm{C} 1 \times 2 \mathrm{C}$ & 0.351 \\
\hline $\mathrm{C} 1 \times 2 \mathrm{~F}$ & 0.800 \\
\hline $\mathrm{C} 2 \times 1 \mathrm{C}$ & 0.282 \\
\hline $\mathrm{C} 2 \times 1 \mathrm{~F}$ & 0.800 \\
\hline
\end{tabular}

$\mathrm{A} 2 \times 1 \mathrm{C}$

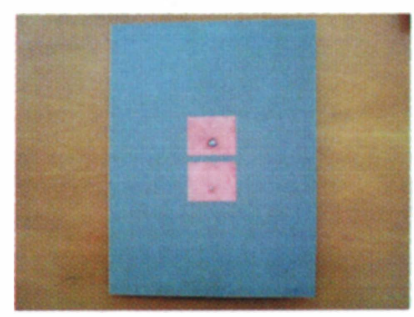

$\mathrm{A} 1 \times 2 \mathrm{C}$

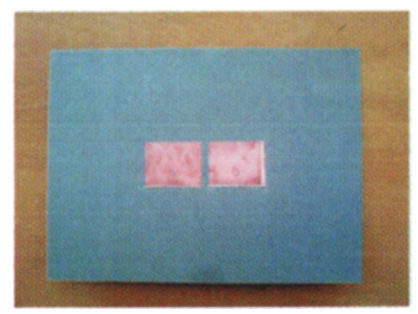

Figure 5. Four different array configurations on substrate A. coaxial switches, and two $5 \mathrm{~m}$ long phase-stable cables with SMA connectors were utilized.

The measurement environment is sketched in Figure 7. It was located at the corner of an empty room with dimensions $16.5 \mathrm{~m}$ by $14.5 \mathrm{~m}$. The locations of the transmitting and receiving arrays were fixed: the distance between them was $3.66 \mathrm{~m}$. The line of sight between them was blocked by a box, which was covered by an aluminum folio. The use of aluminum here was to obtain a conductive obstacle, namely to increase the reflections in the environment. There were three other covered boxes in the environment, along with a chair, a table, and the vector network analyzer placed on the table, as well.

SISO, SIMO, and MIMO measurements were done for the array configurations given in Table 3 . The switches were controlled by the parallel port of the vector network analyzer, by applying $3.3 \mathrm{~V}$ to one of the transistor-transistor logic (TTL) inputs of the switch, which needed at least $2.5 \mathrm{~V}$ to alter state. Furthermore, the transistors required a $12 \mathrm{~V}$ biasing voltage to operate, which was applied by a dc source. One measurement process was formed by 1000 successive realizations, and corresponding channel responses (i.e., $S_{21}$ values) were measured through a 201-point frequency sweep, between 1.75 and $2.25 \mathrm{GHz}$, and saved.

Figure 8 illustrates the average channel coefficients over 1000 different measurements in the SISO cases for all three afore-

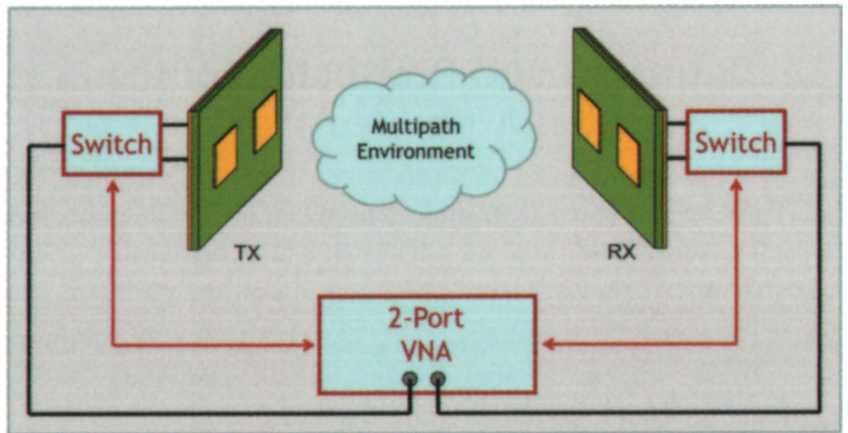

Figure 6. A schematic representation of the indoor MIMO measurement setup.

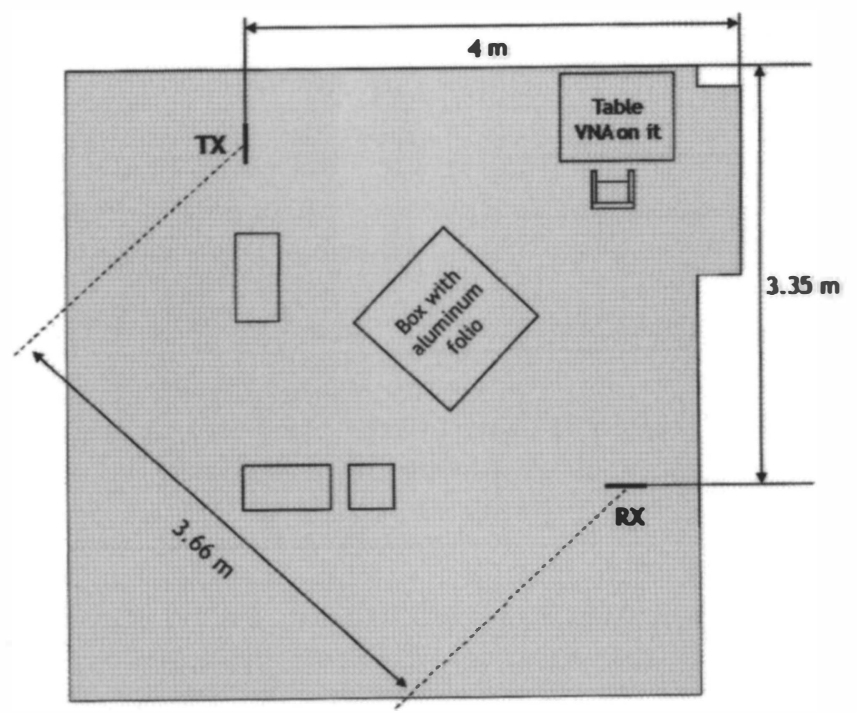

Figure 7. A sketch of the environment. other. Table 3 explicitly shows the configurations of the fabricated arrays. In Table $3, \Delta$ denotes the distance between the feed points of the antenna elements in an array. Note that for the closer element configurations, the patches were physically located apart by $0.05 \lambda$. The distance between the feed points was therefore different for each substrate, since the patch sizes were different. Moreover, three single antennas on each substrate were fabricated to be the transmitting antenna (i.e., $\mathrm{A} 1 \times 1, \mathrm{~B} 1 \times 1, \mathrm{Cl} \times 1$ ) for the singleinput single-output (SISO), and single-input multiple-output (SIMO), cases.

Two different configurations were manufactured for the receiving array: (i) $\mathrm{Al} \times 1$ for the SISO case, and (ii) $\mathrm{A} 1 \times 2 \mathrm{~F}$ for the SIMO and MIMO cases. Briefly, 17 different patch-antenna/array configurations were fabricated. Four of these configurations are shown in Figure 5.

An indoor measurement setup was established at the Antenna Laboratory of Bilkent University. The schematic representation of the setup is given in Figure 6. The channel-matrix entries were measured with a two-port vector network analyzer, together with the appropriate switches as explained in [10]. Two $50 \Omega$ matched 


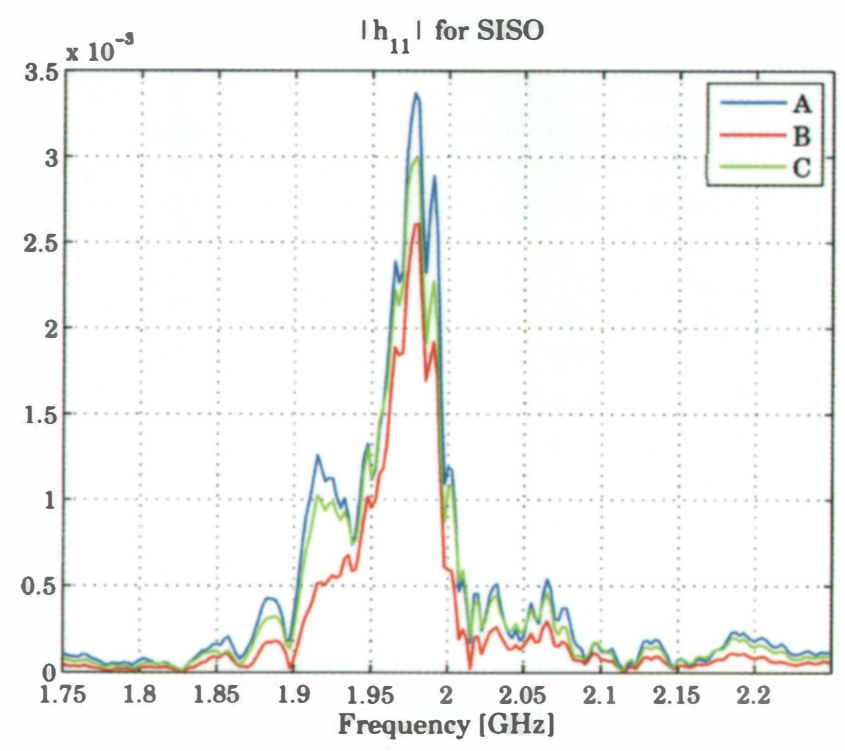

Figure 8. The average channel coefficients over 1000 different measurements in the SISO cases for three different transmitter-array configurations.

(a) SISO I $h_{11} \mid$ for Antenna A

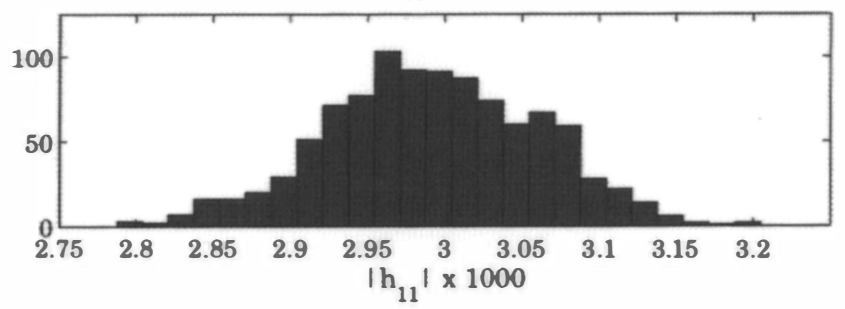

(b) SISO $\angle h_{11}$ for Antenna $A$

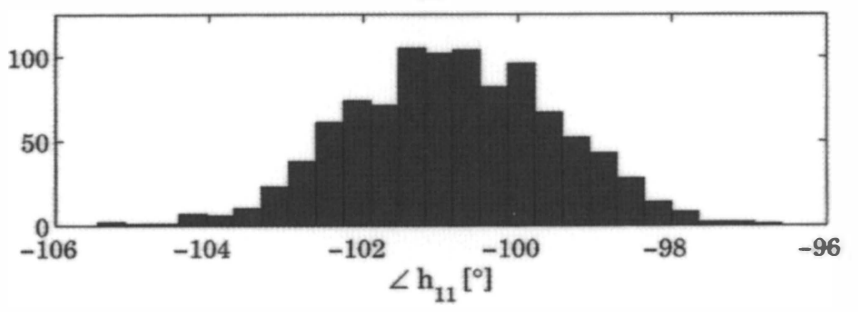

Figure 9. The histograms of the measured channel response at the operating frequency $(1.9725 \mathrm{GHz})$ for Antenna A: (a) magnitude, (b) phase.

mentioned antennas. The narrowband channel could be clearly seen around $1.9725 \mathrm{GHz}$ from the figure. One could expect that the highest capacity would rise from Antenna A, since the average channel response was the highest in magnitude.

The histograms of the magnitude and the phase of the channel response at the operating frequency $(1.9725 \mathrm{GHz})$ for Antenna A are given in Figures $9 \mathrm{a}$ and $9 \mathrm{~b}$, respectively. Note that because there existed neither moving scatterers nor mobile antennas in the environment; in theory, there should have been no variation in the channel capacity. However, very low variations were observed, most probably due to the inherently unpredictable fluctuations in the readings of the vector network analyzer.

Taking the inverse Fourier transform of the channel coefficients, the response could be converted to the time domain. This resulted in a bandlimited version of the channel impulse response, $h(t, \tau)$, where $t$ denotes the time and $\tau$ is the delay component. As mentioned, the channel measured here was time-invariant, because of the absence of moving scatterers or mobile antennas. Hence, the average power delay profile (PDP) can be obtained as

$$
P(\tau)=\frac{1}{1000} \sum_{r=1}^{1000}|h(r, \tau)|^{2},
$$

where $r$ represents one of the 1000 realizations (or time instances). The normalized average power delay profiles for Antennas A, B, and $C$ are plotted in Figure 10. Note that the power results greater than a threshold value are plotted. The threshold was taken as $-25 \mathrm{~dB}$, which is the value the power seemed to contribute before the line-of-sight path $(\tau=12.2 \mathrm{~ns})$. Investigating the figure, one could observe that there were two clusters of scatterers, one composed of paths with delays between 14 and $45 \mathrm{~ns}$, whereas the paths in the other cluster had delay components between 45 and $56 \mathrm{~ns}$. Furthermore, the exponential decay of the power (linear on the $\mathrm{dB}$ scale) both within the clusters and between the maxima of the envelopes could be noticed, which was consistent with [14].

The mean excess delay (the mean delay time), which is the first moment of the given profile, can be expressed as

$$
\langle\tau\rangle=\frac{\sum_{k} P\left(\tau_{k}\right) \tau_{k}}{\sum_{k} P\left(\tau_{k}\right)} .
$$

The mean square excess delay is given by

$$
\left\langle\tau^{2}\right\rangle=\frac{\sum_{k} P\left(\tau_{k}\right) \tau_{k}^{2}}{\sum_{k} P\left(\tau_{k}\right)} .
$$

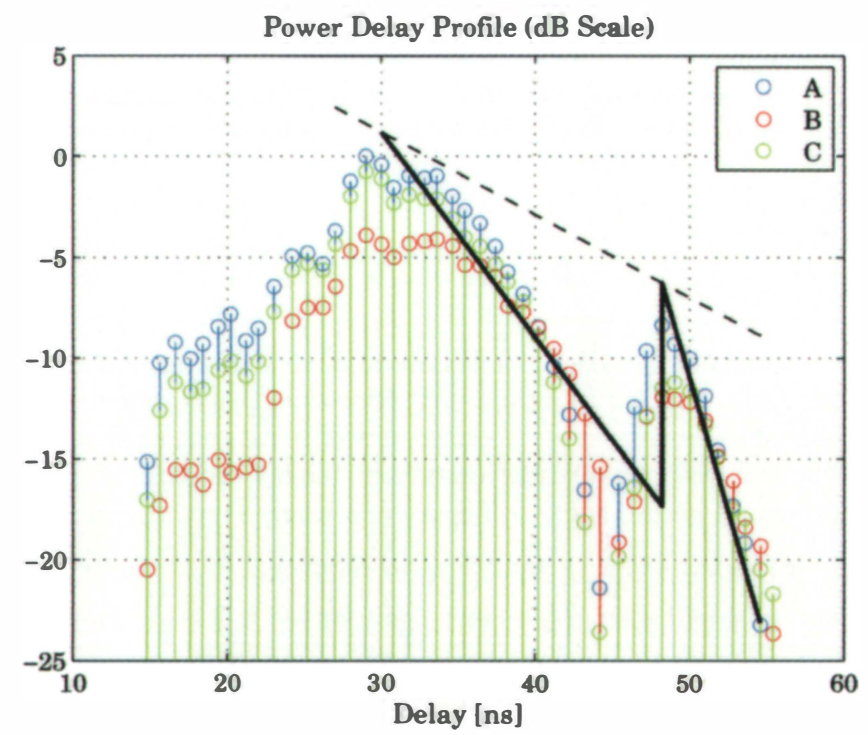

Figure 10. The normalized average power delay profiles of measurements for Antennas A, B, and C. The solid black lines represent the exponential decays in the two clusters of scatterers, whereas the dashed line represents the general exponential decay of the power as a function of delay. 
Table 4. The mean excess delay $(\langle\tau\rangle)$ and rms delay spread $\left(\sigma_{\tau}\right)$ values obtained from the measurements of three antennas.

\begin{tabular}{|c|c|c|c|}
\hline Antenna & A & B & C \\
\hline$\langle\tau\rangle(\mathrm{ns})$ & 31.2 & 33.0 & 31.2 \\
\hline$\sigma_{\tau}(\mathrm{ns})$ & 6.9 & 7 & 6.5 \\
\hline
\end{tabular}

Finally, the standard deviation of the power delay profile gives the root-mean-square (rms) delay spread:

$$
\sigma_{\tau}=\sqrt{\left\langle\tau^{2}\right\rangle-\langle\tau\rangle^{2}}
$$

Table 4 shows the mean delay time and rms delay-spread values obtained from the measurements of the three antennas. Note that these delays were measured relative to the first detectable signal arriving at the receiver, which was not necessarily the largest signal [15]. Note also that although all three antennas were placed in the same location and orientation in a fixed environment, the power delay profile, the mean excess delay, and the rms delay spread values were different because of the different transmission characteristics, such as the radiation intensities of the antennas. It could therefore be concluded that the channel was antenna dependent as well as its response, time-dispersion parameters, and capacity.

\section{Channel Model with Electric Fields (MEF) for Patch Antenna Arrays}

Our channel model with electric fields in [9] used a Galerkinbased MoM solution of the electric-field integral equation, in which the currents on the antenna elements (i.e., free-standing or printed dipoles) were modeled with a single piecewise-sinusoidal basis function. However, in this study, we modified our full-wave channel model by hybridizing it with the commercial electromagnetic solver, such that the MoM solution of the port currents for the probe-fed patch antennas (in the presence of the multipath environment) as well as the radiated fields from the antennas were obtained from the solver. The accuracy was thereby improved.

\subsection{SISO Case}

Consider the SISO wireless communication system shown in Figure 11. The transmitting and receiving patch antennas are assumed to be attached to a vector network analyzer, as mentioned in the previous sections. Between them, the $p$ th propagation path is represented by the angle of departure (AoD), $\Omega_{D, p}$; the angle of arrival (AoA), $\Omega_{A, p}$; the delay component, $\tau_{p}$; and the $2 \times 2$ cross-polarized scattering-coefficient matrix, $\mathbf{A}_{p}$. Path angles are taken from both the elevation and azimuthal planes to create a three-dimensional (3D) multipath environment. Namely, $\left(\Theta_{D}, \Psi_{D},\right)$ and $\left(\Theta_{A}, \Psi_{A}\right)$ pairs form the angle of departure and angle of arrival, respectively. The elevation angles are represented by $\Theta$, whereas the azimuthal angles are shown by $\Psi$. As illustrated in Figure 11, these angles are different from the conventional spherical-coordinate angles $(\theta, \phi)$, though all are convertible to each other via simple trigonometric relations. Note that these parameters are drawn randomly from the corresponding probability density functions, which will be given in the following sections. It should also be noted that this multipath model can involve not only single-bounce scattering, but also multi-bounce scattering as well, since there is no restriction that the departing and arriving paths intersect at one point.

The circuit models in Figure 12 wee used for the transmitting and receiving antennas. In Figure $12, Z_{S}$ and $Z_{L}$ are the source impedance of the transmitting antenna and the load impedance of the receiving antenna, respectively, and are $50 \Omega . Z_{i n}^{t x}$ and $Z_{i n}^{r x}$ represent the impedances recorded by the vector network analyzer, seen through the transmitting- and receiving-antenna ports, respectively. $V^{t x}$ is the source voltage of the transmitting antenna. $V^{o c}$ is the induced (or open-circuit) voltage on the receiving antenna. Finally, $V^{r x}$ is the received voltage recorded on $Z_{L}$. Similar to [9], when $V^{t x}=1 \mathrm{~V}$, the channel response is given by

$$
h=V^{r x},
$$

where

$$
V^{r x}=I^{r x} Z_{L}=\frac{V^{o c}}{Z_{i n}^{r x}+Z_{L}},
$$

and $V^{o c}$ in Equation (20) is obtained as ([16])

$$
V^{o c}=\bar{E}^{r x} \bar{l}^{e}=E_{\theta}^{r x} l_{\theta}^{e}+E_{\phi}^{r x} l_{\phi}^{e}
$$

In Equation (21), $\bar{E}^{r x}$ is the total electric field on the receiving element, and $\bar{l}^{e}$ is its vector effective length [16]. Note that $\bar{E}^{r x}$ is the sum of the fields incident through multipaths and their reflection from the ground plane beneath the substrate. The expression for $\bar{E}^{r x}$ is given by

$$
E_{\theta}^{r x}=\sum_{p}\left(1-R_{\theta, p}\right) E_{p, \theta}^{i n c}\left(\theta_{p}^{r}, \phi_{p}^{r}\right),
$$
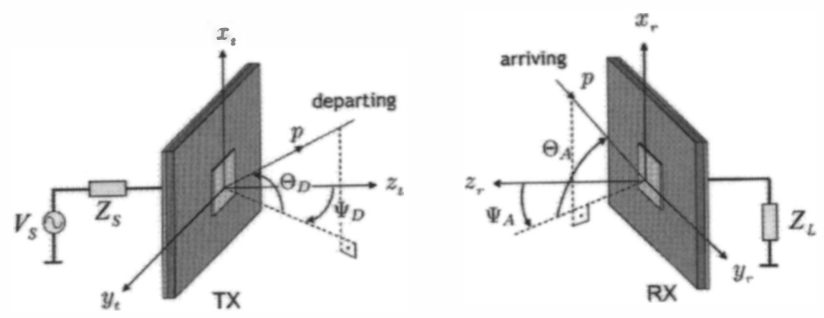

Figure 11. A SISO wireless communication system where the transmitting and receiving patch antennas are attached to a vector network analyzer.
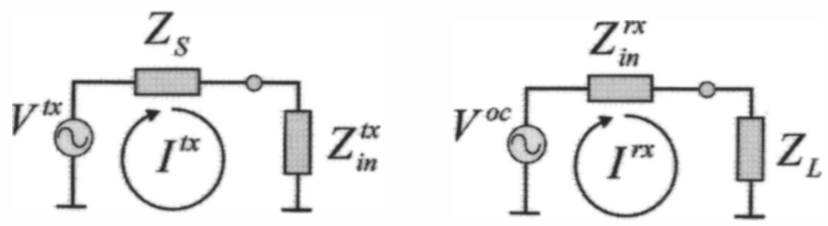

Figure 12. The circuit models for patch antennas at the transmitter (left) and receiver (right). 


$$
E_{\phi}^{r x}=\sum_{p}\left(1+R_{\phi, p}\right) E_{p, \phi}^{i n c}\left(\theta_{p}^{r}, \phi_{p}^{r}\right)
$$

where $E_{p, \phi}^{i n c}\left(\theta_{p}^{r}, \phi_{p}^{r}\right)$ are the field components incident on the receiving antenna arriving through the $p$ th path, corresponding to spherical-coordinate angles that are $\left(\theta_{p}^{r}, \phi_{p}^{r}\right)$, derived from the randomly generated $\Omega_{A, p} \cdot R_{\theta, p}$ and $R_{\phi, p}$ are the reflection coefficients due to the field polarizations, expressions for which are given as follows [17]:

$$
\begin{aligned}
& R_{\theta, p}=\frac{k_{d} \cos \theta_{p}^{r}-j z_{d} \gamma_{p}^{r} \tan \left(\gamma_{p}^{r} d\right)}{k_{d} \cos \theta_{p}^{r}+j z_{d} \gamma_{p}^{r} \tan \left(\gamma_{p}^{r} d\right)}, \\
& R_{\phi, p}=\frac{k_{d} \cos \theta_{p}^{r}+j y_{d} \gamma_{p}^{r} \cot \left(\gamma_{p}^{r} d\right)}{k_{d} \cos \theta_{p}^{r}-j y_{d} \gamma_{p}^{r} \cot \left(\gamma_{p}^{r} d\right)},
\end{aligned}
$$

with

$$
\begin{aligned}
& y_{d}=\frac{1}{z_{d}}=\sqrt{\varepsilon_{r}}, \\
& \gamma_{p}^{r}=\sqrt{k_{d}^{2}-k^{2} \sin ^{2} \theta_{p}^{r}},
\end{aligned}
$$

and

$$
k_{d}=\sqrt{\varepsilon_{r}} k .
$$

From the definition of the vector effective length in [16], $\bar{l}^{e}$ can be written for the receiving antenna as follows:

$$
\bar{l}^{e}\left(\theta_{p}^{r}, \phi_{p}^{r}\right)=\frac{\bar{E}_{r a d}^{r x}\left(\theta_{p}^{r}, \phi_{p}^{r}\right)}{-j k 30 I_{\text {in }}} \frac{R}{e^{-j k R}},
$$

where $\bar{E}_{\text {rad }}^{r x}$ is the radiated field of the receiving antenna when a current $I_{\text {in }}$ is applied to its port as input, and $R$ is the distance from the receiving antenna.

The field arriving through the $p$ th path, $\bar{E}_{p}^{i n c}\left(\theta_{p}^{r}, \phi_{p}^{r}\right)$, can be written in terms of the radiated fields by the transmitting patch antenna $\left(\bar{E}^{t x}\right)$ as

$$
\bar{E}_{p}^{i n c}\left(\theta_{p}^{r}, \phi_{p}^{r}\right)=\mathbf{A}_{p} \bar{E}_{p}^{t x}\left(\theta_{p}^{t}, \phi_{p}^{t}\right) e^{-j k c \tau_{p}},
$$

where

$$
\mathbf{A}_{p}=\left[\begin{array}{cc}
\alpha_{p}^{\theta \theta} & \alpha_{p}^{\theta \phi} \\
\alpha_{p}^{\phi \theta} & \alpha_{p}^{\phi \phi}
\end{array}\right]
$$

is the $2 \times 2$ scattering coefficient matrix of the $p$ th scatterer, which is assumed to be an isotropic radiator. The entries of $\mathbf{A}_{p}$ are modeled as independent and identically distributed (i.i.d.) Gaussian random variables with zero mean and unit variance [9]. Hence, Equation (30) can be rewritten as

$$
\left[\begin{array}{c}
E_{p, \theta}^{i n c} \\
E_{p, \phi}^{i n c}
\end{array}\right]=\left[\begin{array}{cc}
\alpha_{p}^{\theta \theta} & \alpha_{p}^{\theta \phi} \\
\alpha_{p}^{\phi \theta} & \alpha_{p}^{\phi \phi}
\end{array}\right]\left[\begin{array}{c}
E_{p, \theta}^{t x} \\
E_{p, \phi}^{t x}
\end{array}\right] e^{-j k c \tau_{p}},
$$

where $\left(\theta_{p}^{t}, \phi_{p}^{t}\right)$ are the spherical-coordinate angles of the departure, linked with the $p$ th path, derived from the randomly generated $\Omega_{D, p}$, and $c \tau_{p}$ is the total length due to the same path.

Note that the fields radiated by the transmitting patch antenna under the condition $V^{t x}=1 \mathrm{~V}$ are supposed to be obtained from the aforementioned solver. However, because the solver produces the radiated fields for the condition $I^{\text {in }}=1 \mathrm{~A}$, the transmitted fields are expressed in terms of the radiated fields of the solver, $\bar{E}_{s o l}^{t x}\left(\theta_{p}^{t}, \phi_{p}^{t}\right)$, as

$$
\bar{E}^{t x}\left(\theta_{p}^{t}, \phi_{p}^{t}\right)=I^{t x} \bar{E}_{s o l}^{t x}\left(\theta_{p}^{t}, \phi_{p}^{t}\right)=\frac{\bar{E}_{s o l}^{t x}\left(\theta_{p}^{t}, \phi_{p}^{t}\right)}{Z_{\text {in }}^{t x}+Z_{S}} .
$$

In arriving at Equation (33), the circuit model for transmitter given in Figure 12 is used. Equations (20)-(33) relate the source voltage of a transmitting patch antenna to the voltage received by another patch antenna, and, are hence fully capable of obtaining the channel response, i.e., Equation (19), for the SISO case.

\subsection{Multiple Patches at Receiver}

Inspecting Figure 13, let the electric field incident on the two-element patch array at the receiver be $\bar{E}^{\text {inc }}$. This creates the currents, $I_{1,2}^{r x}$, at the ports of the receiving array, along with the fields reflected from the ground plane. Let us denote the radiated field by this array, when its antennas are excited by input currents $I_{1}$ and $I_{2}$, with $\bar{E}_{\text {rad }}^{r x}\left(I_{1}, I_{2}\right)$. Utilizing the superposition principle, this field can be rewritten as

$$
\bar{E}_{\text {rad }}^{r x}\left(I_{1}, I_{2}\right)=I_{1} \bar{E}_{\text {rad }}^{r x}(1,0)+I_{2} \bar{E}_{\text {rad }}^{r x}(0,1),
$$

where $\bar{E}_{\text {rad }}^{r x}(1,0)$ and $\bar{E}_{\text {rad }}^{r x}(0,1)$ are the complex active element field patterns for the first and second patches, respectively. Note that the active element fields are obtained from the solver as well,

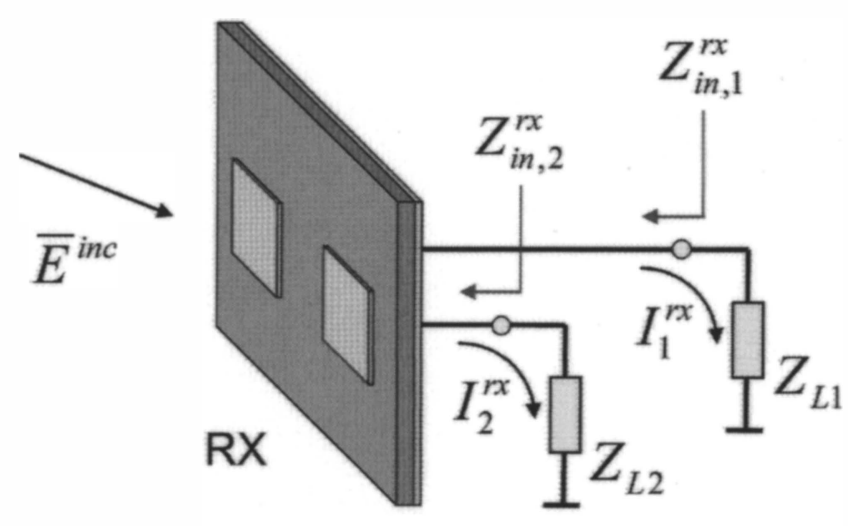

Figure 13. Multiple antennas at the receiver side. 


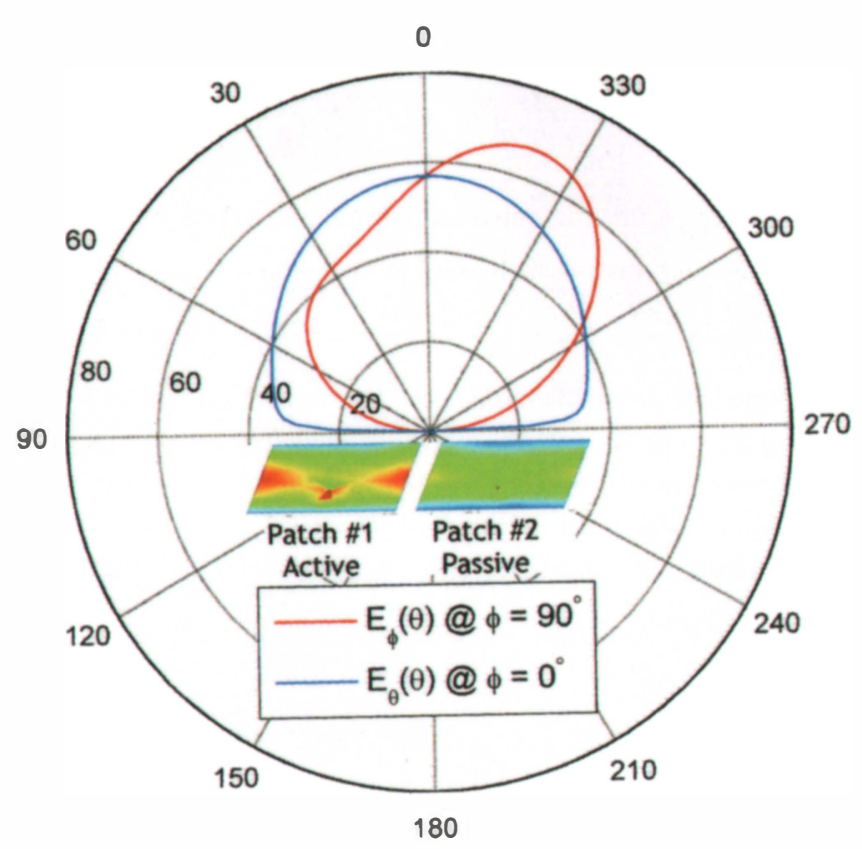

Figure 14. The active element patterns.

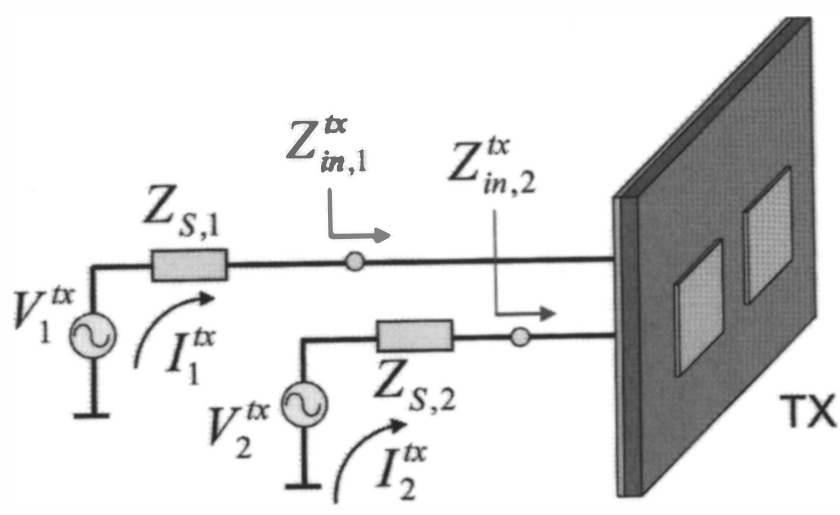

Figure 15. Multiple antennas at the transmitter side.

and typical sample patterns are plotted in Figure 14 for the receiving array of two-patch elements considered in this work. It should be noted that in the course of the calculation of the active element pattern of one patch antenna, the other port is assumed to be terminated by $Z_{L}=50 \Omega$, as in the case of measurements done by the vector network analyzer along with the switch operation.

Making use of the vector effective length definition given in Equation (29), the open-circuit voltages induced on the receiving patch elements can be expressed by

$$
\begin{aligned}
& V_{1}^{o c}=\frac{j}{30 k} \bar{E}_{\text {rad }}^{r x}(1,0)\left[\hat{a}_{\theta} E_{\theta}^{i n c}\left(1-R_{\theta}\right)+\hat{a}_{\phi} E_{\phi}^{i n c}\left(1+R_{\phi}\right)\right], \\
& V_{2}^{o c}=\frac{j}{30 k} \bar{E}_{\text {rad }}^{r x}(0,1)\left[\hat{a}_{\theta} E_{\theta}^{i n c}\left(1-R_{\theta}\right)+\hat{a}_{\phi} E_{\phi}^{i n c}\left(1+R_{\phi}\right)\right] .
\end{aligned}
$$

Note that the open-circuit voltage expressions in Equations (35) and (36) already include the mutual-coupling effects due to the active element patterns. The use of the circuit model given in Figure 12 is therefore adequate to evaluate the received voltages, i.e.,

$$
V_{m}^{r x}=I_{m}^{r x} Z_{L, m}=\frac{V_{m}^{o c}}{Z_{i n, m}^{r x}+Z_{L, m}} Z_{L, m},
$$

where $Z_{L, m}=50 \Omega$ are the termination impedances with $m=1,2$. $Z_{i n, m}^{r x}$ is the input impedance seen through the $m$ th antenna port by the vector network analyzer in the presence of the $n$th patch antenna, with $n \neq m$, whilst the $n$th port is terminated by $50 \Omega$.

In the SIMO case, relating the field incident upon the receiving array to the electric fields radiated by the transmitting patch antenna is then just the same as in Equations (32) and (33). The $2 \times 1$ channel-response matrix entries can be expressed as

$$
h_{m 1}=\frac{V_{m}^{r x}}{V^{t x}}=V_{m}^{r x}
$$

since the transmitting patch antenna is activated by $V^{t x}=1 \mathrm{~V}$.

\subsection{Multiple Patches at the Transmitter}

Because we can evaluate the active element field patterns of given patch-antenna arrays, we may make use of these to obtain the fields radiated by the transmitting array with mutual coupling, in the direction of the $p$ th departing path, as well. Consider Figure 15 for multiple patch antennas at the transmitting side, where $Z_{S, 1}$ and $Z_{S, 2}$ are the source impedances for Patch 1 and Patch 2, respectively, and are set to $50 \Omega$. $Z_{i n, 1}^{t x}$ represents the input impedance seen through the first antenna port by the vector network analyzer in the presence of the second antenna, whilst the second port is terminated by $50 \Omega$ (the same will also be true for $Z_{i n, 2}^{t x}$ when the first port is terminated by $50 \Omega$ ). Applying the aforementioned model-with-electric-fields (MEF) procedure by activating the first antenna with $V_{1}^{t x}=1 \mathrm{~V}$ and $V_{2}^{t x}=0$, the current at the port of the first patch is given by

$$
I_{1}^{t x}=\frac{V_{1}^{t x}}{Z_{i n, 1}^{t x}+Z_{S, 1}}=\frac{1}{Z_{i n, 1}^{t x}+Z_{S, 1}} .
$$

Using this current, the field radiated by the transmitting array in the direction of the $p$ th departing path, $\bar{E}_{p}^{t x}$, can then be written in terms of the active element pattern of the first antenna, $\bar{E}_{p}^{t x}(1,0)$, as

$$
\bar{E}_{p}^{t x}=I_{1}^{t x} \bar{E}_{p}^{t x}(1,0)
$$

Note that during the evaluation of the active element pattern of the first antenna by the solver, $\bar{E}_{p}^{t x}(1,0)$, the port current at the second port is inherently forced to be $I_{2}^{t x}=0$. However, the second antenna still contributes to the radiated field because of the current induced on the passive element due to mutual coupling, as seen in Figure 14.

Relating the transmitted fields to the fields incident upon the receiving array as in Equations (32) and (33), and then the incident fields to the received voltages as in Equations (35)-(37), the chan- 
nel-matrix entries can be evaluated for the first antenna (i.e., $h_{11}$ and $\left.h_{21}\right)$.

Similarly, when the second antenna is activated by $V_{2}^{t x}=1 \mathrm{~V}$ and $V_{1}^{t x}=0$, the field radiated by the transmitting array can be written in terms of the active element pattern of the second antenna, $\bar{E}_{p}^{t x}=\bar{E}_{p}^{t x}(0,1) /\left(Z_{i n, 2}^{t x}+Z_{S, 2}\right)$. Again referring to the corresponding equations that relate the transmitted fields to the received voltages, the channel-matrix entries can be evaluated as

$$
h_{m 2}=\frac{V_{m}^{r x}}{V_{2}^{t x}}=V_{m}^{r x}, m=1,2
$$

for the second antenna.

\section{Experimental and Numerical Results}

\subsection{The Multipath Scenario}

The parameters of the aforementioned three-dimensional multi-bounce scattering environment for the model with electric fields (MEF) are given here. Over $N_{R}=1000$ scenario realizations, all path angles were defined with Laplacian distributions of zero mean and specific angular-spread values $\left(\sigma_{\Theta_{D}}, \sigma_{\Theta_{A}}, \sigma_{\Psi_{D}}\right.$, $\left.\sigma_{\Psi_{A}}\right)$ as

$$
\Phi=-\frac{\sigma}{\sqrt{2}} \operatorname{sgn}(u-0.5) \ln (1-2|u-0.5|)
$$

where $U$ was drawn from the uniform distribution on the unit interval $(0,1]$, and the entries of the cross-polarized scatteringcoefficient matrix were chosen to be independent and identically distributed random variables of zero mean and unit variance. We thus had Laplacian power azimuth/elevation spectra. Numerical values for all parameters for the distributions utilized in this work are given in Table 5. In each channel realization, $S=50$ paths were assumed. Note that the choice of the Laplacian-distributed power azimuth/elevation spectra, as well as the larger azimuthal spread, was consistent with the measurements in the literature [14].

The delay components were drawn randomly from a specific probability distribution function (PDF). The measured power delay profiles previously given for three different antenna types in Figure 10 were averaged (in the linear magnitude scale), and normalized to form a probability distribution function (i.e., the integral of the function over the delay domain gave one). The resulting probability distribution function is plotted in Figure 16a, along with the histogram of generated $N_{R} S$ delay components in Figure 16b. For each of the $N_{R}=1000$ realizations, $S=50$ of these delay components were uniformly chosen and associated with the previously generated angles and scattering matrices to form the corresponding paths.

\subsection{SISO Results}

The capacity result for the $r$ th measurement realization is obtained by
Table 5. The multipath scenario parameters.

\begin{tabular}{|c|c|c|c|}
\hline$\sigma_{\Theta_{D}}$ & $\sigma_{\Theta_{A}}$ & $\sigma_{\Psi_{D}}$ & $\sigma_{\Psi_{A}}$ \\
\hline $25^{\circ}$ & $25^{\circ}$ & $30^{\circ}$ & $30^{\circ}$ \\
\hline
\end{tabular}

(a) PDF

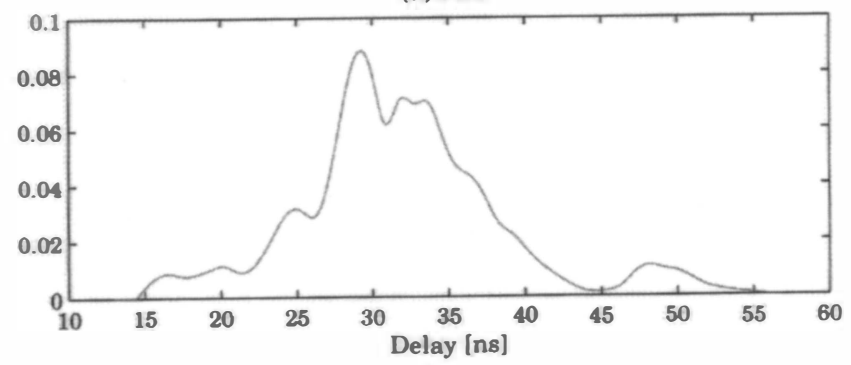

(b) Occurence

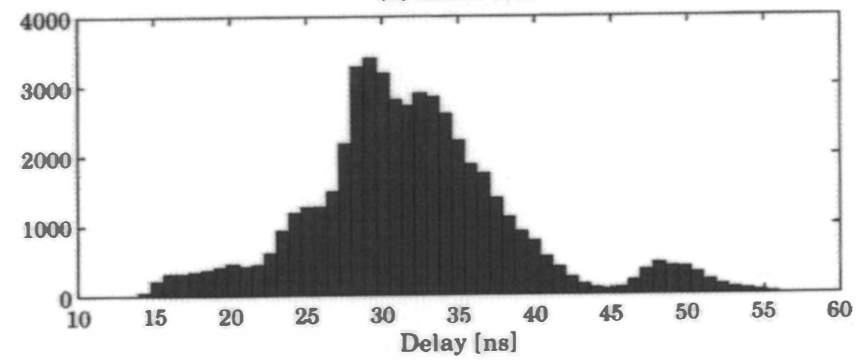

Figure 16. (a) The probability distribution function obtained from the measured power delay profiles; (b) the generated delay components.

$$
C_{r}=\log _{2}\left(\left|\mathbf{I}_{R}+\frac{\rho_{T}}{T} \mathbf{H} \mathbf{H}^{h}\right|\right)
$$

These were averaged over $N_{R}=1000$ total realizations to find the mean capacity results, such as

$$
C=E\left[C_{r}\right]=\frac{1}{N_{R}} \sum_{r=1}^{N_{R}} C_{r} .
$$

$I_{R}$ is the $R \times R$ identity matrix, $|\bullet|$ is the matrix determinant, $\rho_{T}$ is the total transmitted signal-to-noise ratio (SNR), and $(\cdot)^{h}$ and $E[\cdot]$ denote the conjugate-transpose and expectation operations, respectively. Note that $R=T=1$ for the SISO case.

Figure 17 shows the histograms of the measured capacities for three different antenna configurations. The transmitted SNR was adjusted such that the mean capacity of Antenna A was $3 \mathrm{~b} / \mathrm{s} / \mathrm{Hz}$. It was observed that as expected from Figure 8 , Antenna A had a larger capacity than the others.

A comparison of the mean capacities from the model with electric fields (MEF) and measurements is illustrated in Figure 18. Very good agreement was observed between the proposed model with electric fields (MEF) and the measurement results, within 5\% absolute error. Since the substrate upon which Antenna B was located was very thin relative to the other substrates, the structure could be easily bent, and it was difficult to stabilize. The discrepancy for Antenna B may be because of this reason. 


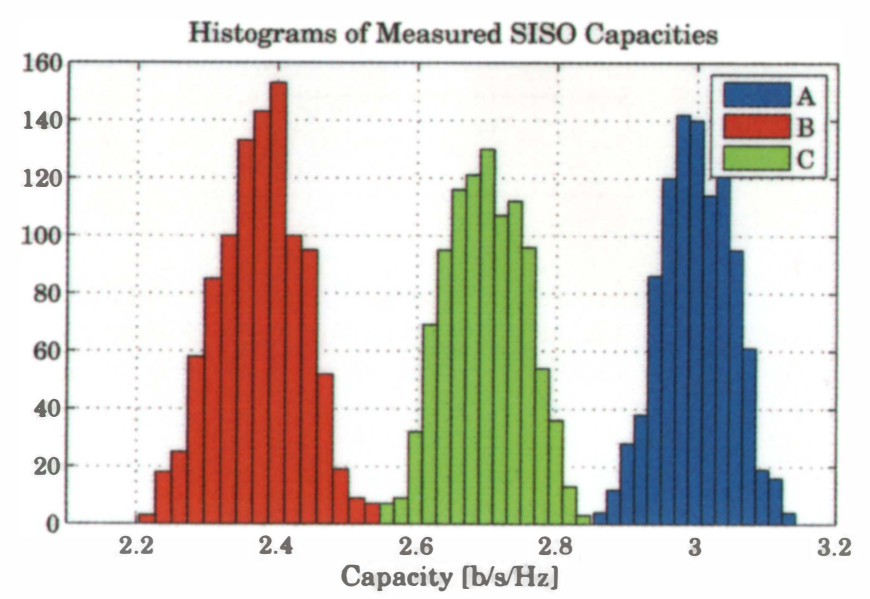

Figure 17. Histograms of the capacities for measurements of three different antenna configurations.

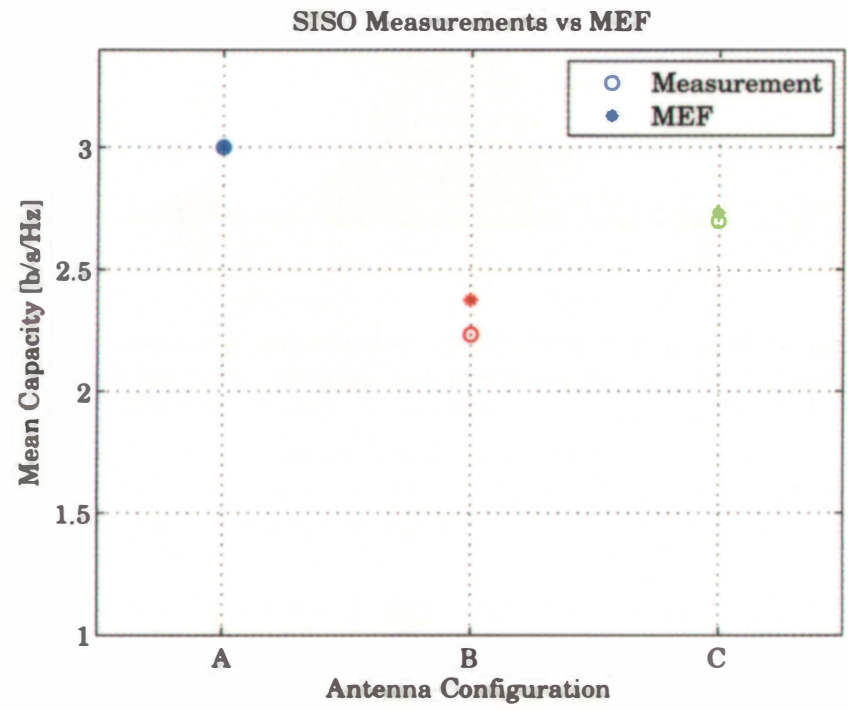

Figure 18. A comparison of the mean capacities obtained from the model with electric fields (MEF) and measurements.

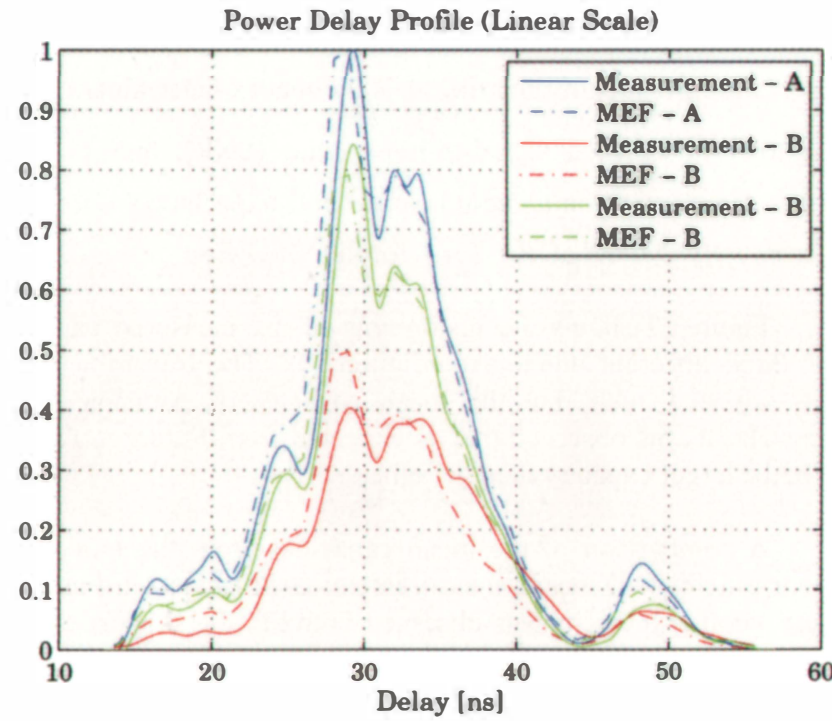

Figure 19. The received power delay profile results obtained from the model with electric fields (MEF) and measurements.
The power delay profile results from the model with electric fields (MEF) and measurements are compared in Figure 19. The normalized received power as a function of delay is plotted in the linear magnitude scale. Again, good agreement was observed.

\subsection{SIMO and MIMO Results}

Note that in the course of the evaluation of capacity values, $R=2$ and $T=1$ in the SIMO case. The receiver array was taken as $\mathrm{A} 1 \times 2 \mathrm{C}$ for all SIMO and MIMO measurements. A comparison of the mean capacities obtained from the model with electric fields (MEF) and measurements is illustrated in Figure 20. Very good agreement was observed between the proposed model with electric fields (MEF) and the measurement results, with less than $6 \%$ absolute error. The mean capacities from the model with electric fields (MEF) and the measurements were next compared for an $R=T=2$ MIMO system. Results are plotted for side-by-side configurations in Figure 21, and for collinear configurations in Fig-

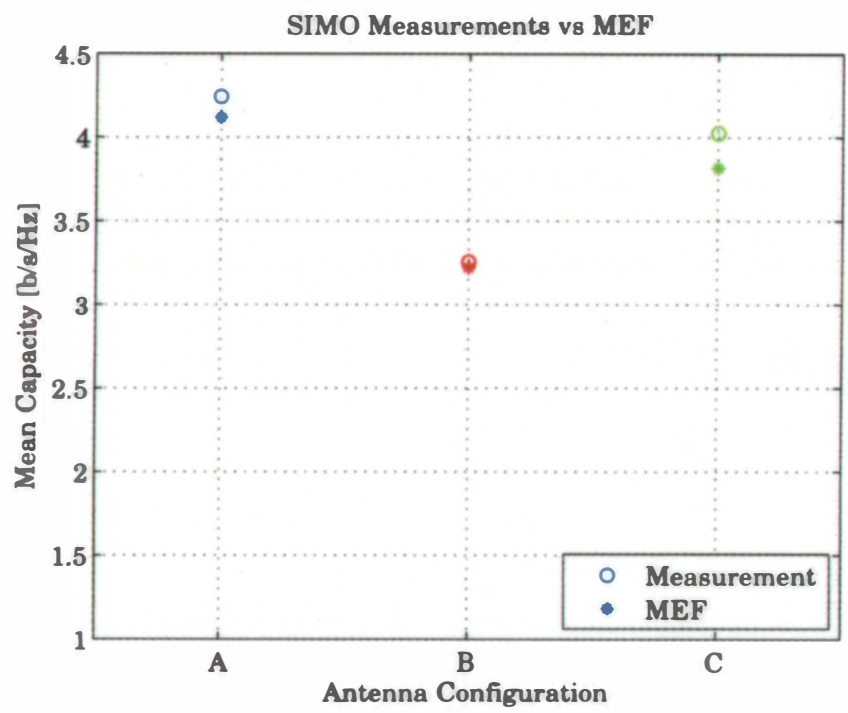

Figure 20. A comparison of the mean capacities from the model with electric fields (MEF) and measurements.

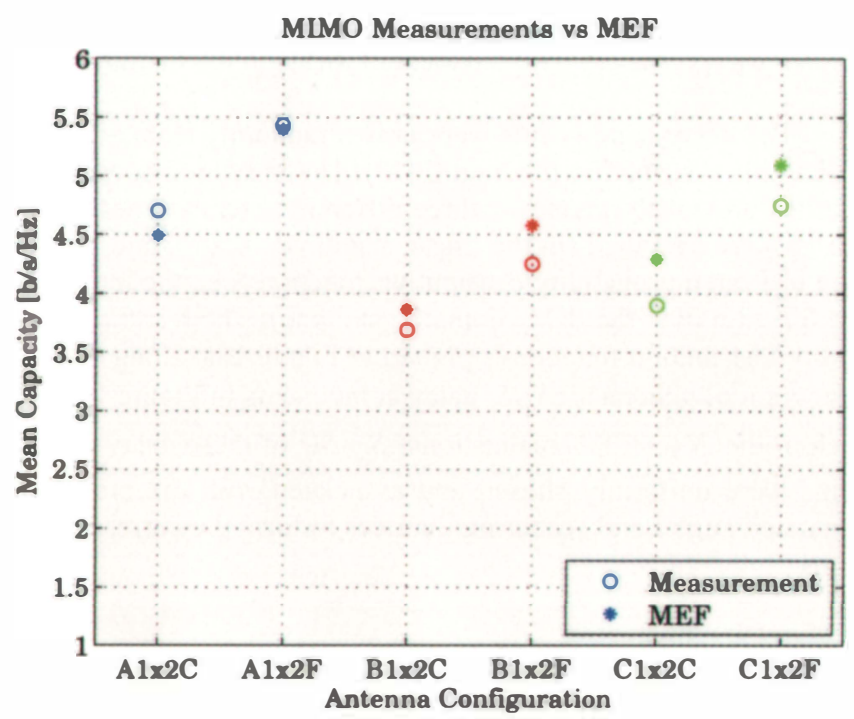

Figure 21. The MIMO capacities from the model with electric fields (MEF) and measurements, for side-by-side patches at the transmitter. 


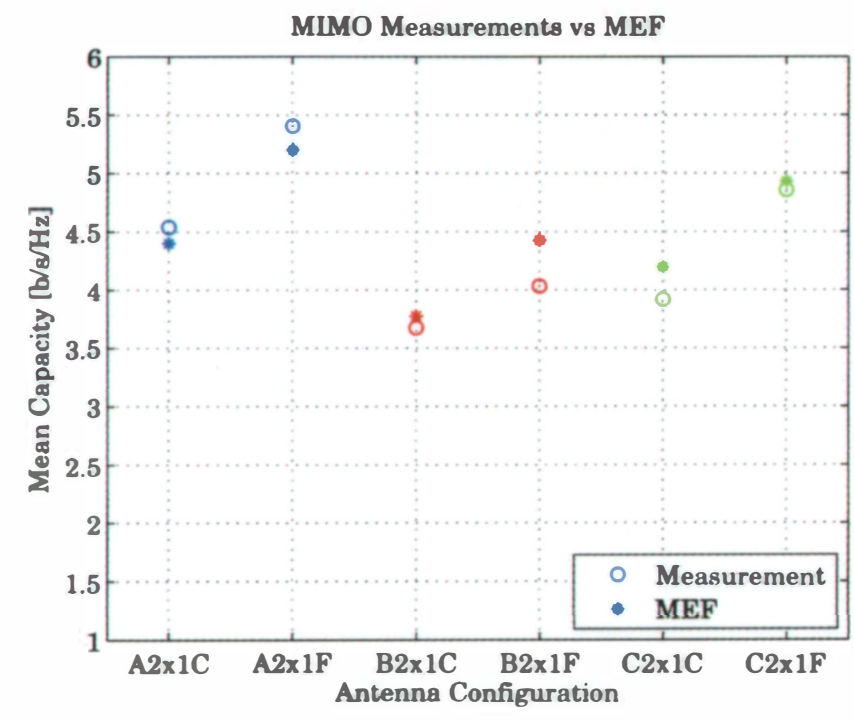

Figure 22. The MIMO capacities from the model with electric fields (MEF) and measurements, for collinear patches at the transmitter.

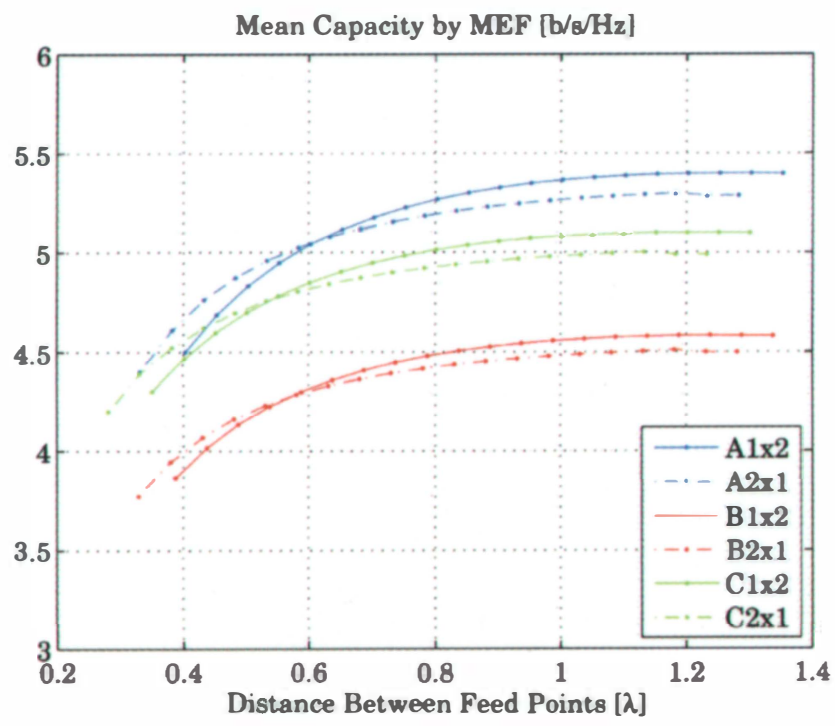

Figure 23. The simulated MIMO capacity results of the sideby-side and collinear arrangements of patch antennas on three different substrates ( $A, B$, and $C)$, as a function of the distance between feed points.

ure 22. Again, good agreement was observed between the proposed model with electric fields (MEF) and the measurement results, with less than $9 \%$ absolute error.

Once the accuracy of the model with electric fields (MEF) was assessed against measurements, it was used to investigate the capacity of patch arrays in detail. Let $\delta=\Delta-W$ denote the distance between the closest edges of the patches of an array. For instance, for $\mathrm{A} 1 \times 2 \mathrm{C}$, the distance between the feed points of the patch elements was given as $\Delta=0.8 \lambda$. However, $\delta$ became $\delta=(0.8-0.353) \lambda=0.477 \lambda$ for this array configuration. We used the model with electric fields (MEF) to evaluate the MIMO capacities of printed patch arrays for 20 different $\delta$ values, ranging from $0.05 \lambda$ to $\lambda$. However, the graphical results are plotted against $\Delta$ because this is the electrically meaningful distance. Fig- ure 23 shows the mean MIMO capacity results of six different patch-array configurations (that is, side-by-side and collinear arrangements of patch antennas on three different substrates, A, B, and $C$ ) as a function of the distance between feed points. Because the substrates $\mathrm{A}$ and $\mathrm{B}$ had similar $\varepsilon_{r}$ values but relatively different thicknesses, comparisons of the corresponding MIMO capacity results presented in Figure 23 might give an idea about the effect of the substrate thickness on the MIMO channel capacity. Similarly, the effect of the dielectric permittivity on the MIMO channel capacity might be deduced from the comparison of the substrate A and $\mathrm{C}$ cases in Figure 23. Similarly to the printed dipole cases presented in [9], as the substrate became thinner, the capacity decreased, since the ground plane was closer to the antenna, and the reflections from it had a destructive effect that can also be explained via image theory. Based on Figures 22 and 23, one could say that the mutual coupling between the patch antennas degrades the channel capacity. It was clearly seen that when the antennas were far away from each other, the channel capacity became higher, due to the weaker mutual coupling between the antennas. On the other hand, when the mean-channel-capacity curves were investigated for side-by-side and collinear configurations in Figure 23, it was observed that the capacity of the collinear arrangements was better for small separations. However, the capacity of the side-by-side arrangements increased faster with increasing separation, and eventually became better. These observations can easily be explained via the mutual coupling between the patches. In the side-by-side configuration, the antennas were in the $\mathrm{H}$ plane of each other, and the dominant mutual coupling mechanism was the space waves. Hence, for small separations, the coupling was strong, resulting in a decrease in the channel capacity. However, coupling through the space waves decays fast with increasing separation and, hence, the channel capacity improves faster. On the other hand, the antennas were in the E plane of each other for the collinear configuration, and the dominant mutual-coupling mechanism was then the surface waves. The coupling was thus weaker than that of the side-by-side configuration for small separations, leading to a slightly higher capacity. However, the coupling decayed slower than that of the side-by-side arrangement for increasing separations, resulting in a smaller capacity for large separations. Nevertheless, the maximum improvement in the capacity was around $1 \mathrm{~b} / \mathrm{s} / \mathrm{Hz}$. From the capacity point of view, antennas can thus be located nearby to each other for the design cases in which the physical size for antenna/array placement is limited.

Finally, as opposed to the printed dipoles [9], increasing $\varepsilon_{r}$ did not increase the capacity for printed patches. This may be because both the electrical and physical dimensions of antennas on substrate B were the smallest dimensions among the others [18]. Reference [18] also stated the reason for less capacity for thinner substrates in the cavity-model context, such that thinner substrates resulted in higher quality factors, which decreased the radiation efficiency as the stored energy was increased.

\section{Conclusions}

The MIMO performance for arrays of printed rectangular patch antennas was analyzed, using a modified version of the fullwave channel model (MEF) given in [9]. The modification was performed by hybridizing the existent procedure with an available commercial electromagnetic solver. Various array configurations were designed, manufactured, and their MIMO performance was measured in an indoor environment. Good agreement was achieved 
between the measurements and simulations using the model with electric fields (MEF). The effects of the mutual coupling and the electrical properties of printed patches on the MIMO capacity were explored.

\section{Acknowledgments}

This work was supported by the Turkish Scientific and Technical Research Agency (TÜBİTAK), under Grants EEEAG106E081, EEEAG-104E044, and EEEAG-105E065. It was supported by the Turkish Academy of Sciences (TÜBA)-GEBIP, and also in part by the European Commission in the framework of the FP7 Network of Excellence in Wireless COMmunications NEWCOM++ (contract n. 216715).

\section{References}

1. J. Guterman, A. A. Moreira, and C. Peixeiro, "Integration of Omnidirectional Wrapped Microstrip Antennas into Laptops," IEEE Antennas and Wireless Propagation Letters, 5, 1, December 2006, pp. 141-144.

2. A. Forenza and R. W. Heath Jr., "Benefit of Pattern Diversity via Two-Element Array of Circular Patch Antennas in Indoor Clustered MIMO Channels," IEEE Transactions on Communications, 54, 5, May 2006, pp. 943- 954.

3. J. W. Wallace, M. A. Jensen, A. Gummalla, and H. B. Lee, "Experimental Characterization of the Outdoor MIMO Wireless Channel Temporal Variation," IEEE Transactions on Vehicular Technology, 56, 3, May 2007, pp. 1041-1049.

4. M. Sanchez-Fernandez, E. Rajo-Iglesias, O. Quevedo-Teruel, and M. L. Pablo-Gonzalez, "Spectral Efficiency in MIMO Systems Using Space and Pattern Diversities Under Compactness Constraints," IEEE Transactions on Vehicular Technology, 57, 3, May 2008, pp. 1637-1645.

5. D. G. Landon and C. M. Furse, "Recovering Handset Diversity and MIMO Capacity with Polarization-Agile Antennas," IEEE Transactions on Antennas and Propagation, 55, 11, November 2007, pp. 3333-3340.

6. Y. Ouyang, D. J. Love, and W. J. Chappell, "Body-Worn Distributed MIMO System," IEEE Transactions on Vehicular Technology, 58, 4, May 2009, pp. 1752-1765.

7. D. Piazza, P. Mookiah, M. D'Amico, and K. R. Dandekar, "Experimental Analysis of Pattern and Polarization Reconfigurable Circular Patch Antennas for MIMO Systems," IEEE Transactions on Vehicular Technology, 59, 5, June 2010, pp. 2352-2362.

8. O. Quevedo-Teruel, M. Sanchez-Fernandez, M. L. PabloGonzalez, and E. Rajo-Iglesias, "Alternating Radiation Patterns to Overcome Angle-of-Arrival Uncertainty," IEEE Antennas and Propagation Magazine, 52, 1, February 2010, pp. 236-242.

9. C. A. Tunc, D. Aktas, V. B. Ertürk, and A. Altintas, "Capacity of Printed Dipole Arrays in MIMO Channel," IEEE Antennas and Propagation Magazine, 50, 5, October 2008, pp. 190-198.

10. M. D. Migliore, D. Pinchera, and F. Schettino, "Improving Channel Capacity Using Adaptive MIMO Antennas," IEEE Trans- actions on Antennas and Propagation, 54, 11, November 2006, pp. 3481-3489.

11. D. Pinchera, J. W. Wallace, M. D. Migliore, and M. A. Jensen, "Experimental Analysis of a Wideband Adaptive-MIMO Antenna," IEEE Transactions on Antennas and Propagation, 56, 3, March 2008, pp. 908-913.

12. A. Street, L. Lukama, and D. Edwards, "Use of VNAs for Wideband Propagation Measurements," IEE Proceedings - Communications, 148, 6, December 2001, pp. 411-415.

13. F. Abboud, J. Damiano, and A. Papiernik, "Simple Model for the Input Impedance of Coax-Fed Rectangular Microstrip Patch Antenna for CAD," IEE Proceedings H Microwaves, Antennas and Propagation, 135, 5, October 1988, pp. 323-326.

14. R. Vaughan and J. B. Andersen, Channels, Propagation and Antennas for Mobile Communications, London, IEE Press, 2003.

15. T. S. Rappaport, Wireless Communications: Principles \& Practice, Upper Saddle River, NJ, Prentice Hall, 1996.

16. C. A. Balanis, Antenna Theory: Analysis and Design, Third Edition, New York, John Wiley \& Sons, 2005.

17. W. C. Chew, Waves and Fields in Inhomogeneous Media, Piscataway, IEEE Press, 1995.

18. Y. T. Lo and S. W. Lee, Antenna Handbook: Theory, Applications and Design, Berlin, Springer, 1988.

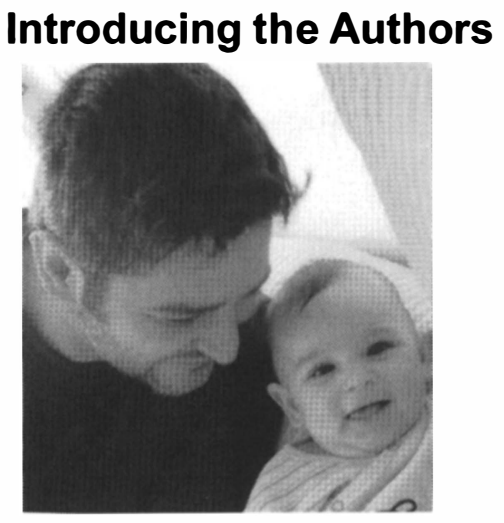

Celal Alp Tunc received the BSc degree in Electronics and Communication Engineering from Istanbul Technical University, Istanbul, in 2001, and his MSc and PhD degrees in Electrical and Electronics Engineering from Bilkent University, Ankara, in 2003 and 2009, respectively. Between 2001 and 2009, he worked as a teaching and research assistant at Bilkent University, Electrical and Electronics Engineering Department. He then served the Turkish Armed Forces as a tank-team commander with the rank of second lieutenant. He is pursuing research and development activities at Artlab Ltd. (Ankara Research and Technology Laboratory), of which he is a co-founder. His research areas are numerical techniques for computational electromagnetics, large-scale electromagnetic radiation-scattering problems, antenna analysis and propagation-channel modeling for MIMO wireless communications, design and production of microstrip MIMO antenna arrays, MIMO channel measurements, optimization techniques, and algorithms for electromagnetic/information theoretic problems. Dr. Tunc was a recipient of the Young Scientist Award of URSI in 2008. He is a congress member of Besiktas JK. Dr. Tunc is married to Ilknur, and father of Duru. 


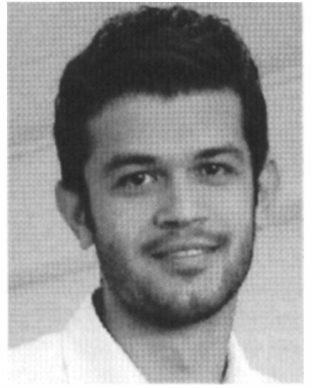

Ugur Olgun was born in 1986, in Tokat, Turkey. He received his BS degree in Electrical and Electronics Engineering from Bilkent University, Ankara, Turkey, in 2008, and is currently working toward the $\mathrm{PhD}$ degree at The Ohio State University, Columbus, Ohio. He is currently a Graduate Research Associate with the ElectroScience Laboratory, The Ohio State University. His research interests include applied EM theory, antennas, nonlinear circuit matching, RFIDs, and wireless power transfer. $\mathrm{Mr}$. Olgun was the recipient of the Undergraduate Scholarship presented by the IEEE Microwave Theory and Techniques Society (IEEE MTT-S) in 2008. In addition, Ugur received the first-place award at the 2010 John D. and Alice Nelson Kraus Memorial Student Poster Competition, and a third-place student paper award at the 2010 Antenna Measurements and Techniques Association (AMTA) conference.

Vakur B. Ertürk received the BS degree in Electrical Engineering from the Middle East Technical University, Ankara, Turkey, in 1993, and the MS and PhD degrees from The Ohio-State University (OSU), Columbus, in 1996 and 2000, respectively. He is currently an Associate Professor with the Electrical and Electronics Engineering Department, Bilkent University, Ankara. His research interests include the analysis and design of planar and conformal arrays, active integrated antennas, scattering from and propagation over large terrain profiles, and metamaterials. Dr. Ertürk served as the Secretary/Treasurer of IEEE Turkey Section as well as the Turkey Chapter of the IEEE Antennas and Propagation, Microwave Theory and Techniques, Electron Devices, and Electromagnetic Compatibility Societies. He was the recipient of a 2005 URSI Young Scientist Award, and a 2007 Turkish Academy of Sciences Distinguished Young Scientist Award.

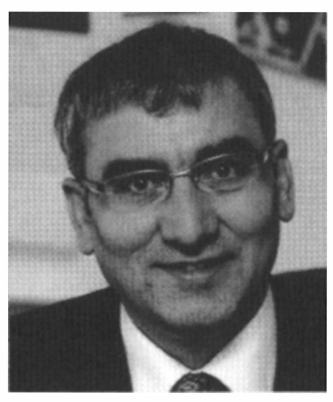

Ayhan Altintas received the BS and MS degrees from Middle East Technical University, Ankara, Turkey, and the PhD degree from Ohio State University, all in Electrical Engineering in 1979, 1981, and 1986, respectively. Currently, he is Professor and Chair of Electrical Engineering at Bilkent University, Ankara, Turkey. He is also the Director of the Communication and Spectrum Management Research Center (ISYAM). Dr. Altintas was the Chair of the IEEE Turkey Section for 1991-1993 and 1995-1997. $\mathrm{He}$ is the founder and first Chair of the IEEE AP/MTT Chapter in the Turkey Section. At present, he is the National Chair of URSI Commission B (Scattering and Diffraction). His research interests are in electromagnetics, antennas, propagation, and wireless-communication systems. Dr. Altintas was a Fulbright Scholar, and an Alexander von Humboldt Fellow. He received the Ohio State University ElectroScience Laboratory Outstanding Dissertation Award of 1986, the IEEE 1991 Outstanding Student Branch Counselor Award, the 1991 Research Award of the Prof. Mustafa N. Parlar foundation of METU, and the Young Scientist Award of the Scientific and Technical Research Council of Turkey (Tubitak) in 1996. He is a member of Sigma Xi and Phi Kappa Phi, and a recipient of the IEEE Third Millennium Medal. (4: 